\title{
Incompressible relativistic spheres: Electrically charged stars, compactness bounds, and quasiblack hole configurations
}

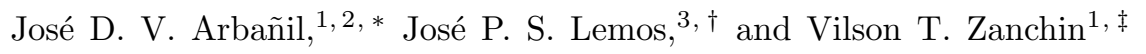 \\ ${ }^{1}$ Centro de Ciências Naturais e Humanas, Universidade Federal do ABC, \\ Rua Santa Adélia 166, 09210-170 Santo André, SP, Brazil. \\ ${ }^{2}$ Centro de Ciências Tecnológicas, Universidade do Estado de Santa Catarina, \\ Rua Paulo Malschitzki S/N, 89219-710 Joinville, SC, Brazil. \\ ${ }^{3}$ Centro Multidisciplinar de Astrofísica - CENTRA, Departamento de Física, \\ Instituto Superior Técnico - IST, Universidade de Lisboa - UL, \\ Avenida Rovisco Pais 1, 1049-001 Lisboa, Portugal.
}

\begin{abstract}
We investigate the properties of relativistic star spheres made of an electrically charged incompressible fluid, generalizing, thus, the Schwarzschild interior solution. The investigation is carried by integrating numerically the hydrostatic equilibrium equation, i.e., the Tolman-Oppenheimer-Volkoff (TOV) equation, with the hypothesis that the charge distribution is proportional to the energy density. We match the interior to a Reissner-Nordström exterior, and study some features of these star spheres such as the total mass $M$, the radius $R$, and the total charge $Q$. We also display the pressure profile. For star spheres made of a perfect fluid there is the Buchdahl bound, $R / M \geq 9 / 4$, a compactness bound found from generic principles. For the Schwarzschild interior solution there is also the known compactness limit, the interior Schwarzschild limit where the configurations attain infinite central pressure, given by $R / M=9 / 4$, yielding an instance where the Buchdahl bound is saturated. We study this limit of infinite central pressure for the electrically charged stars and compare it with the Buchdahl-Andréasson bound, a limit that, like the Buchdahl bound for the uncharged case, is obtained by imposing some generic physical conditions on charged configurations. We show that the electrical interior Schwarzschild limit of all but two configurations is always below the Buchdahl-Andréasson limit, i.e., we find that the electrical interior Schwarzschild limit does not generically saturate the Buchdahl-Andréasson bound. We also find that the quasiblack hole limit, i.e., the extremal most compact limit for charged incompressible stars, is reached when the matter is highly charged and the star's central pressure tends to infinity. This is one of the two instances where the Buchdahl-Andréasson bound is saturated, the other being the uncharged, interior Schwarzschild solution.
\end{abstract}

\section{INTRODUCTION}

The search for matter field solutions in general relativity started with Schwarzschild [1] who studied a perfect fluid with spherically symmetric energy density distribution given by $\rho(r)$ and pressure $p(r)$, where $r$ is the radial coordinate, for a constant energy equation of state, $\rho=\rho_{0}$, with $\rho_{0}$ a constant, matched to a static spherically symmetric vacuum exterior spacetime. The exterior solution is the Schwarzschild solution, while the whole solution, comprised of the interior plus exterior, is called the Schwarzschild interior solution 11. Schwarzschild also obtained a compactness limit, the interior Schwarzschild limit. He showed [1] that at $R / M=9 / 4$ the central pressure becomes infinite, and for smaller $R$ there is no central pressure that can sustain the configuration, and it presumably collapses, where $R$ is the radius of the configuration and $M$ its mass. Tolman [2] and Oppenheimer and Volkoff [3] then discussed in detail the structure of those matter field equations by writing the pressure gradient field in a convenient form, now called the Tolman-Oppenheimer-Volkoff

\footnotetext{
*Electronic address: dfi6jdav@joinville.udesc.br

${ }^{\dagger}$ Electronic address: joselemos@ist.utl.pt

${ }^{\ddagger}$ Electronic address: zanchin@ufabc.edu.br
}

(TOV) equation. Volkoff 4 and then Misner in his lectures [5] showed how the Schwarzschild interior solution can be extracted directly from the TOV equation and rederived the limit of $R / M=9 / 4$ as had been found by Schwarzschild [1]. Nowadays, standard texts in general relativity exhibit the Schwarzschild interior solution and its limit. A constant density equation of state for a fluid in a star is certainly interesting not only from a historical perspective. Notably, a constant density is achieved when the matter is negligibly compressible and can sustain huge pressures. This happens when the velocities of the matter particles become relativistic, i.e., when the temperatures are of the order of the particles' rest mass if the matter is composed of bosons, or when the Fermi levels are again of the order of the particles' rest mass if the matter is composed of fermions. This means that in both cases the densities approach one particle per cubic Compton wavelength. In addition, it is from an incompressible equation of state that one extracts the important compactness Schwarzschild limit, which can then be compared with other compactness bounds, and the results give a clean and robust picture. Of course, an incompressible equation of state also has some drawbacks, as the speed of sound through such a medium is infinite, although this does not have a major influence in the overall structure. There are many other equations of state that can be used to study compact stars. Indeed, for instance, Oppenheimer and Volkoff [3] applied 
the method to study compact neutron stars, stars that do not obey an incompressible equation of state. As expected then, the most compact neutron stars are not as compact as the interior Schwarzschild limit allows.

Buchdahl [6] took further interest in general relativistic equilibrium and proved that, under certain conditions, a spherically symmetric matter configuration could only exist when the radius $R$ of the configuration divided by its mass $M$ satisfies the relation $R / M \geq 9 / 4$. The result is quite general. Indeed the only assumptions for providing this result are that the fluid's energy density $\rho$ is non-negative and decreasing outward, and the pressure $p$ is non-negative and isotropic, i.e., the fluid is perfect, and the boundary defined by $p=0$ is matched to the exterior Schwarzschild solution. The inequality is a compactness bound, the Buchdahl bound, and the value $R / M=9 / 4$ is the Buchdahl limit. It is a limit of limits. It also means a star made of such a fluid cannot approach its own gravitational radius $R / M=2$ and so trapped surfaces are eliminated. Although derived from completely different means, the interior Schwarzschild limit (i.e., the limit of infinite central pressure for incompressible spheres) [1] and the Buchdahl limit (i.e., the limit for perfect fluids obeying reasonable physical conditions) 6] coincide, and so the interior Schwarzschild solution with infinite pressure saturates the Buchdahl bound and, thus, realizes the Buchdahl limit.

The interest of adding electric charge to the matter in writing the TOV equation was understood by Bekenstein in 7. Indeed, although a significant excess of electric charge in stars is unlikely, its study in stars is of great interest since it mimics other fields and possible alterations in the gravitational field as proposed in alternative theories of gravity. Perhaps, the most simple electric star is a star with a Schwarzschild incompressible interior with some type of added electric charge distribution. Adding electric charge to these configurations can bring some insight to their overall structure in more complex situations. Thus, in this spirit and sticking to incompressible fluids, de Felice and collaborators [8, 9] studied the structure of electric stars with a power-law charge distribution $q(r)$ of the form $q(r)=Q(r / R)^{n}$, for some exponent $n \geq 3$, total charge $Q$, and star's radius $R$, matching the solutions, at the star's boundary, to the ReissnerNordström solution for the given star's mass $M$, radius $R$, and charge $Q$. Numerical techniques were used. They also analyzed the most compact stars for each $Q$. Anninos and Rothman [10, also studied incompressible fluids with somewhat more intricate charge distributions. Now, in the case the radius $R$ of the charged star is taken to the gravitational radius of the star itself, the configuration is called a quasiblack hole. It is an extremal configuration. In this extremal configuration the total electric charge of the star $Q$ is equal to the star's mass $M, Q=M$, and the configuration has a radius $R$ equal to its own gravitational radius $r_{+}=M$, i.e., $R=M$. This configuration is highly compact and is a limit for the case $Q=M$. In [8 10] such configurations were detected. The stability of physical system, in particular of electrically charged stars, is always of importance. An analysis of the stability of such stars against radial perturbations has been performed in 9, 10, with the conclusion that for a range of the star parameters, and depending on how stiff the perturbed matter is, these systems can be stable.

There has also been interest in finding a Buchdahl electric limit formula which started in the works [11, 12]. In [13, among other cases studied, the $\rho=\rho_{0}$ solution, with $\rho_{0}$ a constant, with the $n=3$ power-law distribution for the charge given in [8, 9], was revisited with the aim of finding the most compact configuration and a Buchdahl electric limit formula. Up to now, the sharpest bound has been given by Andréasson [14. This Buchdahl-Andréasson bound for electrically charged matter is taken from the condition $p+2 p_{T} \leq \rho$, where $p$ and $p_{T}$ are the radial and tangential pressures, respectively, and some other reasonable physical conditions 14. It is given by $R / M \geq 9 /\left(1+\sqrt{1+3 Q^{2} / R^{2}}\right)^{2}$. Surprisingly, although derived from completely different hypotheses, the Buchdahl bound for the uncharged $Q=0$ case $R / M \geq 9 / 4$ is reobtained. It also gives the quasiblack hole limit, for $Q=R$ one obtains $R=M$, and so $Q=M$ also. Charged shells saturate the BuchdahlAndréasson bound [14], whereas the $\rho=\rho_{0}$ solution, with $\rho_{0}$ a constant, and $q(r)=Q(r / R)^{3}$ of [13] do not, as noticed in 14. Presumably, the whole set of solutions found in [8, 9] also does not saturate generically the Buchdahl-Andréasson bound.

Motivated by these settings we want to further investigate relativistic stars made of an incompressible fluid, $\rho=\rho_{0}$, with $\rho_{0}$ being a constant. In order to probe the genericity of the results mentioned above we will assume a new charge distribution $q(r)$, namely, we take the electric charge density $\rho_{e}$ as proportional to the energy density, $\rho_{e}=\alpha \rho_{0}$, with $\alpha$ a number obeying $0 \leq \alpha \leq 1$. This is a possible assumption as one might think that the net charge is inherent to each particle, having arisen from some physical consorted process. Then the charged distribution $q(r)$ is taken from the Maxwell field equation $\frac{d q(r)}{d r}=4 \pi \rho_{e}(r) r^{2} \sqrt{A(r)}$, where $A(r)$ is the $g_{r r}(r)$ component of the spacetime metric. This gives a $q(r)$ different from the ones considered in [8 10, namely, $q(r)=Q(r / R)^{n}$ (see also [13]). Other $q(r)$ distributions can be thought of, such as one that has most of the charge at or near the core of the star. Here we stick to $\rho_{e}=\alpha \rho_{0}$. We then analyze the structure of such spheres and study the compactness bounds, namely, we numerically probe the electric interior Schwarzschild limit with an electric charge $Q$ within the range $0 \leq Q \leq M$ and compare the results to the Buchdahl-Andréasson bound 14. We also study the quasiblack hole limit $Q=R=M$ of this set of configurations.

It is worth mentioning that there are equations of state, other than an incompressible one, that can be used in the study of electrically charged stars and that should be mentioned. For instance, Cooperstock and de la Cruz [15] and Florides [16] used an equation of the form $\rho(r)+q^{2}(r) / 8 \pi r^{4}=$ constant and studied some prop- 
erties of the solutions (see also [17]). Polytropic equations were used in [18 21], where star configurations and their structure were studied, and the Schwarzschild electric limit for the given equation of state and for a given charge was considered. There are also works that treat a quark deconfining phase in compact neutron stars [22, where an unbalance of electric or color charge might appear. These works [15,22] stayed away from the extremal quasiblack hole configuration. Works that did consider the Schwarzschild electric limit and the quasiblack hole limit are (i) works that analyzed the Cooperstock-de la Cruz-Florides equation of state [15, 17] as in [23, (ii) works that used a generic polytropic equation of state with any exponent as in 24, (iii) works with a dust equation of state, i.e., where the fluid is composed of purely extremal charged matter, also called electrically counterpoised matter, where static equilibrium configurations at the quasiblack hole limit, with $Q=M$ and $R=M$ were found [25, 26], (iv) works that in addition to the electric charge have a scalar charge [27], and of course (v) works with an incompressible equation of state [8 10] as mentioned before, to name a sample. Note that some works, e.g. 24, have been calling the Buchdahl limit what should in fact be named the interior Schwarzschild limit. Works that treat generically quasiblack holes are [28, 29], for a review see [30].

The paper is organized as follows. In Sec. 2, we give the general relativistic equations, the equations of structure for a static spherically symmetric configuration, the equations of state for the energy density and charge density, and discuss the boundary conditions. In Sec. 3, we study numerically the structure of an electrically charged incompressible star. We give the numerical input values and then find the mass of the relativistic star as a function of the energy density, the radius as a function of the energy density, and the charge as a function of the energy density. The behavior of the mass, radius, and charge of the stars for some central pressures is also shown. In addition, the pressure profile, i.e., $p(r)$, is displayed for some typical stars. In Sec. 4, we present the electrical Schwarzschild limit for these stars and compare with the Buchdahl-Andréasson limit. In Sec. 5 we study in detail the quasiblack hole limit of such a relativistic star and give the behavior of the redshift at the surface of the quasiblack hole as a function of the star's intrinsic parameters. In Sec. 6, we conclude. In the Appendix we give the equations of structure in dimensionless form.

\section{GENERAL RELATIVISTIC EQUATIONS}

\subsection{Basic equations}

We are interested in analyzing the properties of highly compacted charged spheres as described by EinsteinMaxwell equations with charged matter (in this section we put $c=1$ and $G=1$ ), i.e.,

$$
\begin{aligned}
& G_{\mu \nu}=8 \pi T_{\mu \nu}, \\
& \nabla_{\nu} F^{\mu \nu}=4 \pi J^{\mu},
\end{aligned}
$$

where greek indices are spacetime indices running from 0 to 3 , with 0 being a time index. The Einstein tensor $G_{\mu \nu}$ is defined in terms of the Ricci tensor $R_{\mu \nu}$, the metric tensor $g_{\mu \nu}$, and the Ricci scalar $R$ by the well-known relation $G_{\mu \nu}=R_{\mu \nu}-\frac{1}{2} g_{\mu \nu} R$. $T_{\mu \nu}$ stands for the energymomentum tensor, which in this study is written as a sum of two terms,

$$
T_{\mu \nu}=E_{\mu \nu}+M_{\mu \nu} .
$$

The first part $E_{\mu \nu}$ is the electromagnetic energymomentum tensor, which is given in terms of the Faraday-Maxwell tensor $F_{\mu \nu}$ by the relation

$$
E_{\mu \nu}=\frac{1}{4 \pi}\left(F_{\mu}^{\gamma} F_{\nu \gamma}-\frac{1}{4} g_{\mu \nu} F_{\gamma \beta} F^{\gamma \beta}\right) .
$$

The matter energy-momentum content of the spacetime is represented by $M_{\mu \nu}$, which has the form of the energymomentum tensor of a perfect fluid,

$$
M_{\mu \nu}=(\rho+p) U_{\mu} U_{\nu}+p g_{\mu \nu},
$$

with $\rho$ and $p$ being the energy density and the pressure of the fluid, respectively, and $U_{\mu}$ is the fluid fourvelocity. Equation (2) is the Maxwell equation, stating the proportionality between the covariant derivative $\nabla_{\nu}$ of the Faraday-Maxwell tensor $F_{\mu \nu}$ and the electromagnetic four-current $J_{\mu}$. For a charged fluid, this current is given in terms of the electric charge density $\rho_{e}$ by

$$
J^{\mu}=\rho_{e} U^{\mu} .
$$

The other Maxwell equation $\nabla_{[\alpha} F_{\beta \gamma]}=0$, where [...] means antisymmetrization, is automatically satisfied for a properly defined $F_{\mu \nu}$.

\subsection{Equations of structure}

The charged incompressible fluid spheres considered here are described by a static fluid distribution with spherical symmetry, in such a way that the line element is of the form

$$
d s^{2}=-B(r) d t^{2}+A(r) d r^{2}+r^{2}\left(d \theta^{2}+\sin ^{2} \theta d \phi^{2}\right),
$$

where $t, r, \theta$, and $\phi$ are the usual Schwarzschild-like coordinates, and the metric potentials $A(r)$ and $B(r)$ are functions of the radial coordinate $r$ only.

The assumed spherical symmetry of the spacetime implies that the only nonzero components of a purely electrical Faraday-Maxwell tensor $F^{\mu \nu}$ are $F^{t r}=-F^{r t}$, where $F^{t r}$ is a function of the radial coordinate $r$ alone, $F^{t r}=F^{t r}(r)$. The other components of $F^{\mu \nu}$ are identically zero. Hence, the only nonvanishing component of the Maxwell equation (2) is given by

$$
\frac{d q(r)}{d r}=4 \pi \rho_{e}(r) r^{2} \sqrt{A(r)},
$$


with $q(r)=r^{2} \sqrt{A(r) B(r)} F^{t r}(r)$ representing the total electric charge inside a spherical surface labeled by the radial coordinate whose value is $r$. With this, and from the line element (7), the Einstein equation (1) yields the following relevant relations

$$
\begin{aligned}
& \frac{1}{A(r)}\left[1-\frac{r}{A(r)} \frac{d A(r)}{d r}\right]=1-8 \pi r^{2}\left[\rho(r)+\frac{q^{2}(r)}{8 \pi r^{4}}\right] \\
& \frac{1}{A(r)}\left[1+\frac{r}{B(r)} \frac{d B(r)}{d r}\right]=1+8 \pi r^{2}\left[p(r)-\frac{q^{2}(r)}{8 \pi r^{4}}\right]
\end{aligned}
$$

Defining the new quantity $m(r)$ in such a way that

$$
\frac{1}{A(r)}=1-\frac{2 m(r)}{r}+\frac{q^{2}(r)}{r^{2}},
$$

and replacing $A(r)$ from Eq. (11) into Eq. 9 it gives

$$
\frac{d m(r)}{d r}=4 \pi \rho(r) r^{2}+\frac{q(r)}{r}\left[\frac{d q(r)}{d r}\right] .
$$

The new function $m(r)$ represents the gravitational mass inside the sphere of radial coordinate $r$, and Eq. (12) then represents the energy conservation, as measured in the star's frame.

An additional equation is obtained from the contracted Bianchi identity $\nabla_{\mu} T^{\mu \nu}=0$, which gives

$$
\frac{d B(r)}{d r}=\frac{B(r)}{p(r)+\rho(r)}\left[\frac{q(r)}{2 \pi r^{4}} \frac{d q(r)}{d r}-2 \frac{d p(r)}{d r}\right] .
$$

Finally, replacing Eq. (8) and the conservation equation (13) into Eq. (10) it yields

$$
\frac{d p}{d r}=-(p+\rho) \frac{\left[4 \pi p r+\frac{m}{r^{2}}-\frac{q^{2}}{r^{3}}\right]}{\left[1-\frac{2 m}{r}+\frac{q^{2}}{r^{2}}\right]}+\rho_{e} \sqrt{A} \frac{q}{r^{2}},
$$

where to simplify the notation we have dropped the functional dependence, i.e., $A(r)=A, m(r)=m, q(r)=q$, $\rho(r)=\rho, p(r)=p$, and $\rho_{e}(r)=\rho_{e}$. Equation (14) is the TOV equation [2, 3], modified by the inclusion of electric charge [7] (see also [24]).

\subsection{Equation of state and the charge density profile}

In the present model there are six unknown functions, $B(r), m(r), q(r), \rho(r), p(r)$, and $\rho_{e}(r)$, and just four equations, Eqs. (8), 12), (13), and (14). Additional relations are obtained from a model for the matter, i.e., from a model for the cold fluid, which furnishes relations between the pressure and the energy density. For an electrically charged fluid, a relation defining the electric charge distribution is also needed.

For the present analysis we assume an incompressible fluid, i.e.,

$$
\rho(r)=\rho_{0},
$$

with $\rho_{0}=$ constant. So the energy density is constant along the whole star.

Following [18, 19] (see also 21] and 24]), we assume a charge density proportional to the energy density,

$$
\rho_{e}=\alpha \rho_{0}
$$

where, in geometric units, $\alpha$ is a dimensionless constant which we call the charge fraction. The charge density along the whole star is, thus, constant as well.

We have now four equations: Eqs. (8), 12, , 13, and (14); and four unknowns: $B(r), m(r), q(r)$, and $p(r)$, as $\rho_{0}$ and $\rho_{e}$ are given in (15) and (16), respectively. The resulting set of equations constitutes the complete set of structure equations which, with some appropriate boundary conditions, can be solved simultaneously.

\subsection{The boundary conditions and the exterior vacuum region to the star}

The numerical integration of the system of equations is performed along the radial coordinate $r$, from the center toward the surface of the star. The conditions at the center of the star $(r=0)$ are $m(r=0)=0, q(r=0)=0$, $p(r=0)=p_{c}, \rho(r=0)=\rho_{c}, \rho_{e}(r=0)=\rho_{e c}$, and $A(r=0)=1$, where $p_{c}$ is the central pressure, and $\rho_{c}$ and $\rho_{e c}$ are the central energy density and the central charge distributions, respectively, which by assumption are constant throughout the star. The surface of the star is defined by the vanishing of the pressure. Since the pressure decreases outwards, the integration is stopped at the point $r=R$ for which $p(R)=0$. The solution is then matched to the exterior Reissner-Nordström spacetime, with metric given by

$$
d s^{2}=-F(r) d T^{2}+\frac{d r^{2}}{F(r)}+r^{2}\left(d \theta^{2}+\sin ^{2} \theta d \phi^{2}\right),
$$

where

$$
F(r)=1-2 M / r+Q^{2} / r^{2},
$$

with the outer time $T$ being proportional to the inner time $t, M$ and $Q$ being the total mass and the total charge of the star, respectively. The full set of boundary conditions at the surface of the star is $B(R)=1 / A(R)=$ $F(R), m(R)=M, q(R)=Q$, besides $p(R)=0$.

\subsection{Our aim}

Using the whole set of equations and boundary conditions, it is our interest to study the structure of these incompressible charged stars. Given values for the energy density $\rho_{0}$ and for the charge fraction $\alpha$, we can analyze the system for different values of central pressure $p_{c}$. For each $\alpha$, and thus for each $Q / M$, there is a set of star solutions, each corresponding to a given $p_{c}$. The limiting solution that has $p_{c}=\infty$ gives the interior Schwarzschild limit. We then compare this interior 
Schwarzschild limit with the electric Buchdahl limit, or Buchdahl-Andréasson limit [14, i.e., the minimum ratio for $R / M$ for a given $Q / M$. In addition, the most extreme configuration, the one that has $Q / M=1$, has as the limiting solution the quasiblack hole, i.e., a star whose boundary is located at its own gravitational radius. We look in detail into these solutions and to their corresponding features.

\section{THE STRUCTURE OF A CHARGED INCOMPRESSIBLE RELATIVISTIC STAR SPHERE AND THE BUCHDAHL LIMIT}

\subsection{Numerical input values}

We now analyze the structure of incompressible charged star spheres with $\rho_{0}=$ constant and $\rho_{e}=\alpha \rho_{0}=$ constant. The set of coupled equations, Eqs. (8), (12), (13), 14, (15), and (16), and the boundary conditions adopted at the center are integrated up to the boundary of the star. The integration is performed upon putting the equations in a dimensionless form (see the Appendix). For each given energy density $\rho_{0}$, charge fraction $\alpha$ and central pressure $p_{c}$, the system of equations is numerically solved using a fourth order Runge-Kutta method.

Here we are going to analyze the numerical results and to plot a few graphs showing several parameters of the stars, such as the radius, the gravitational mass, the total charge, and some other interesting quantities. In order to obtain the corresponding values in appropriate units, it is convenient to restore the gravitational constant $G$, while keeping the speed of light set to unity, i.e., in this section, we use units such that $G=7.42611 \times 10^{-28}[\mathrm{~m} / \mathrm{kg}]$ and $c=1$.

For ease of comparison with other works, we consider the energy density and the central pressure in the ranges $1.0 \times 10^{13}\left[\mathrm{~kg} / \mathrm{m}^{3}\right] \leq \rho_{0} \leq 1.0 \times 10^{20}\left[\mathrm{~kg} / \mathrm{m}^{3}\right]$ and $1.0 \times 10^{13}\left[\mathrm{~kg} / \mathrm{m}^{3}\right] \leq p_{c} \leq 1.0 \times 10^{18}\left[\mathrm{~kg} / \mathrm{m}^{3}\right]$, respectively. The interval of energy densities considered here covers the range of values of the central energy density of real neutron stars. On the other hand, the values considered for the central pressures are within the range of the central pressures considered in [19.

Let us last mention that the charge fraction $\alpha$ is varied in the interval $0 \leq \alpha \leq 1$. However, it is important to say that, in the intervals of energy density and central pressure considered in the present work, the value $\alpha=$ 0.99 is in fact the largest value of the charge fraction we have used in the numerical analysis. This value was chosen for comparison to our previous work [24]. For larger values of $\alpha$ the numerical calculations become very slow and eventually fail to converge for $\alpha$ very close to unity. We have not found equilibrium solutions for $\alpha>1$.

The star's mass $M$, its radius $R$, and total charge $Q$, are found when the pressure at the surface of the object is equal to zero $p(r=R)=0$. We present the main features of these three quantities in the following subsections.
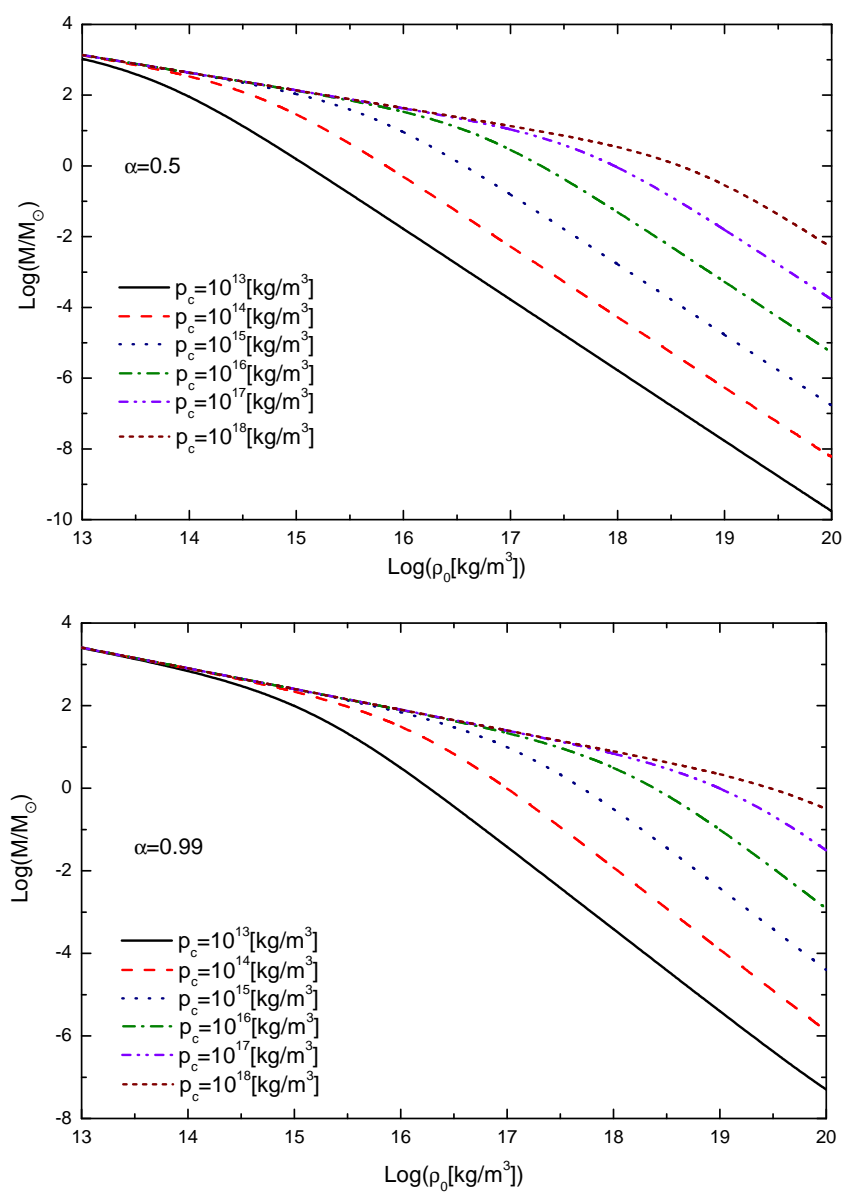

FIG. 1: The ratio $M / M_{\odot}$ of the incompressible charged spheres as a function of the energy density $\rho_{0}$ for six values of the central pressure $p_{c}$ as indicated. The top panel is for $\alpha=0.5$ and the bottom panel for $\alpha=0.99$.

\subsection{Mass of a charged relativistic incompressible star as a function of the energy density}

The mass of the star (in units of solar masses $M_{\odot}$ ) as a function of the energy density $\rho_{0}$ is shown in Fig. 1 for some values of the central pressure. The top panel gives the curves for the charge fraction $\alpha=0.5$ and the bottom panel for $\alpha=0.99$. The values of the energy density and central pressure are in the ranges $1.0 \times 10^{13}$ to $1.0 \times 10^{20}\left[\mathrm{~kg} / \mathrm{m}^{3}\right]$ and $1.0 \times 10^{13}$ to $1.0 \times 10^{18}\left[\mathrm{~kg} / \mathrm{m}^{3}\right]$, respectively. In all the presented cases, we can note that the mass of the star decreases monotonically with the increase of the energy density, and in a larger rate for small pressures than for large pressures. This can be understood in the sense that stars with large energy densities have to have less mass in order for equilibrium to be maintained. In addition, we can see that, for low densities, large changes in the central pressure $p_{c}$ do not imply large changes in the mass, on the contrary, the mass changes very little. It signals the fact that, for low densities, the increase on the central pressure is very sensitive to small increases in the star's mass. Notice also that for 


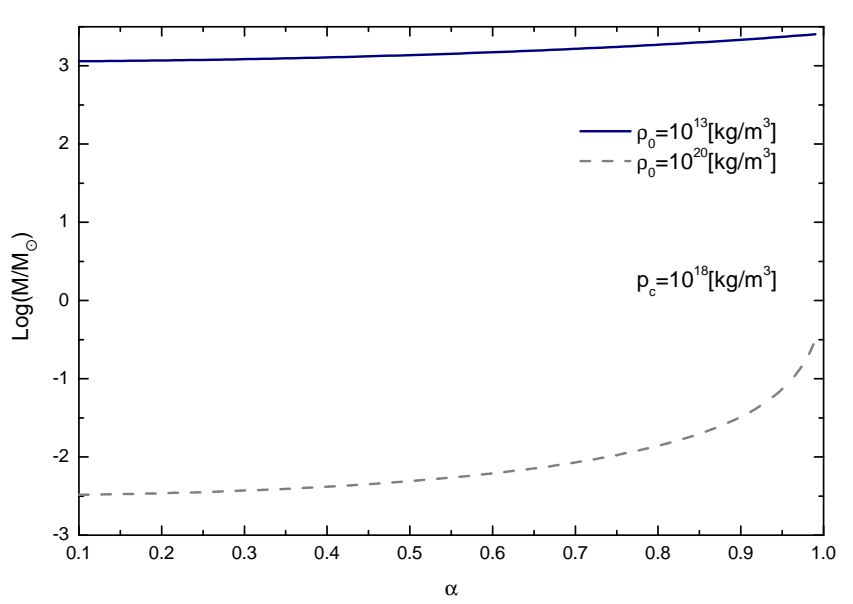

FIG. 2: The ratio $M / M_{\odot}$ of the incompressible charged spheres as a function of the charge fraction $\alpha$ for the central pressure $p_{c}=1.0 \times 10^{18}\left[\mathrm{~kg} / \mathrm{m}^{3}\right]$ and two values of the energy density $\rho_{0}$, namely, $1.0 \times 10^{13}\left[\mathrm{~kg} / \mathrm{m}^{3}\right]$ and $1.0 \times 10^{20}\left[\mathrm{~kg} / \mathrm{m}^{3}\right]$.

high densities and small central pressures the masses of the related incompressible stars are very small compared to the case of high densities and large central pressures.

Figure 2 shows the dependence of the mass of the object in units of solar masses as a function of the charge fraction $\alpha$ for two values of the energy density $\rho_{0}$ as indicated. The general feature is that the mass grows monotonically with $\alpha$, and faster for higher densities. Without going into great detail, let us comment on the change in the mass of an incompressible charged sphere when the charge fraction $\alpha$ changes from 0.5 to 0.99 . For $p_{c}=1.0 \times 10^{18}\left[\mathrm{~kg} / \mathrm{m}^{3}\right]$ and $\rho_{0}=1.0 \times 10^{13}\left[\mathrm{~kg} / \mathrm{m}^{3}\right]$, the increase in the mass is about $84.8029 \%$, whereas for the same $p_{c}$ but with $\rho_{0}=1.0 \times 10^{20}\left[\mathrm{~kg} / \mathrm{m}^{3}\right]$ the increase in the mass is about $6220.67 \%$. From these examples, it can be deduced that for low densities, in comparison with large densities, the mass of the object does not change considerably with the increment of the charge fraction $\alpha$.

\subsection{Radius of a charged relativistic incompressible star as a function of the energy density}

The radius of the star as a function of the energy density is shown in Fig. 3 where we plot the ratio $R / M$ against the energy density $\rho_{0}$ for some values of the central pressure $p_{c}$. The energy density and central pressure values in the figure are the same as in Fig. 11, so the values of the considered energy densities are in the range $1.0 \times 10^{13}$ to $1.0 \times 10^{20}\left[\mathrm{~kg} / \mathrm{m}^{3}\right]$. Two values of the charge fraction were taken for comparison, $\alpha=0.5$ (top panel) and $\alpha=0.99$ (bottom panel). In all curves, the relation $R / M$ grows with $\rho_{0}$, indicating that less compact stars are found for large energy densities. On the other hand, we see that there is also an influence of $p_{c}$ in the degree of compaction of a star. The larger $p_{c}$ is, the smaller the value of $R / M$. This is of course expected, as the star
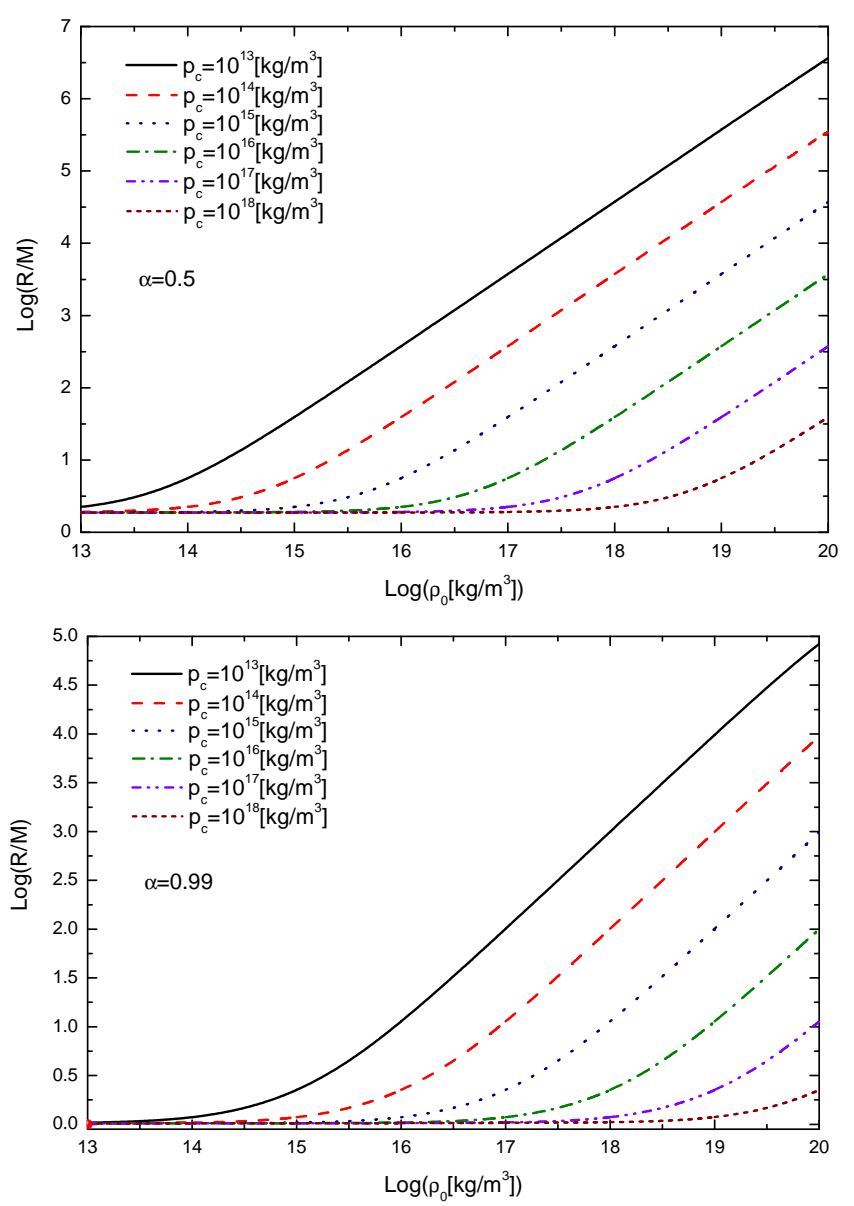

FIG. 3: The ratio $R / M$ of the incompressible charged spheres as a function of the energy density $\rho_{0}$ for six values of the central pressure $p_{c}$, as indicated. The top panel is for $\alpha=$ 0.5 and the bottom panel for $\alpha=0.99$. The red point in the bottom panel indicates the quasiblack hole configuration where $R / M \simeq 1.0$.

gets more compact, the gravitational action is stronger and there is a need for a greater pressure throughout the star, including the central pressure $p_{c}$, to counterbalance.

In Fig. 4, the dependence of $R / M$ is shown as a function of the charge fraction $\alpha$ for the minimum and the maximum considered energy densities, i.e., $\rho_{0}=1.0 \times$ $10^{13}\left[\mathrm{~kg} / \mathrm{m}^{3}\right]$ and $\rho_{0}=1.0 \times 10^{20}\left[\mathrm{~kg} / \mathrm{m}^{3}\right]$, respectively, and for the central pressure $p_{c}=1.0 \times 10^{18}\left[\mathrm{~kg} / \mathrm{m}^{3}\right]$. The ratio $R / M$ decreases with the charge fraction, meaning that the stars bearing a large amount of charge are more compact than the stars with little or no charge. The case that has a central pressure $p_{c}$ much higher than the energy density $\rho_{0}$, the case shown in the full line, is the case that approximates well the electric interior Schwarzschild limit for each charge fraction $\alpha$. So, for $\alpha=0.0$, one gets $R / M \simeq 2.25$ which is the original interior Schwarzschild limit $R / M=9 / 4$ [1]. For the case of $\alpha=0.99$, i.e., the extremal charge case, we obtain $R / M \simeq 1.0$ which is in accord with the limits set by Andréasson for charged spheres [14. In this case the limit is a quasiblack hole, the red point in the figure. 


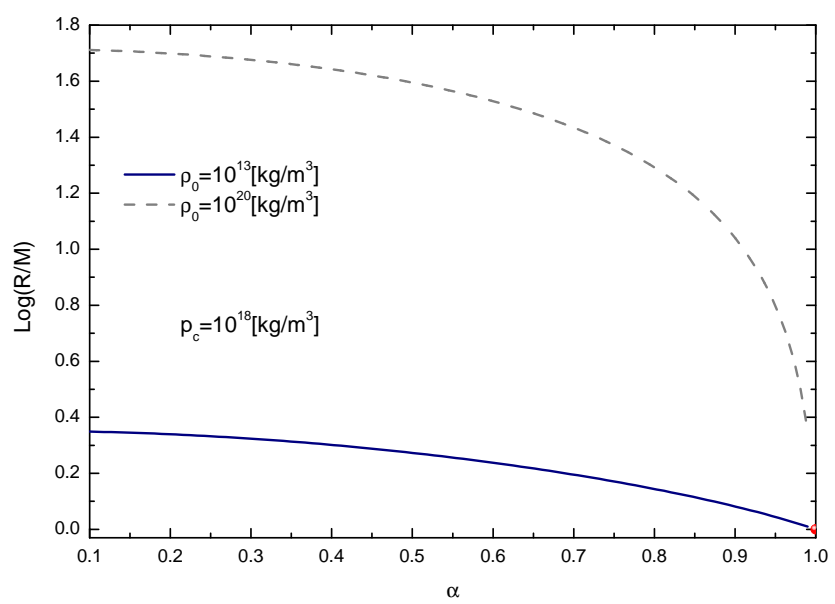

FIG. 4: The ratio $R / M$ of the incompressible charged spheres as a function of the charge fraction $\alpha$ for a central pressure $p_{c}=1.0 \times 10^{18}\left[\mathrm{~kg} / \mathrm{m}^{3}\right]$ and two values of the energy density, one case with $\rho_{0}=1.0 \times 10^{20}\left[\mathrm{~kg} / \mathrm{m}^{3}\right]>p_{c}$, and the other case with $\rho_{0}=1.0 \times 10^{12}\left[\mathrm{~kg} / \mathrm{m}^{3}\right]<<p_{c}$, as indicated. The red point in the figure indicates the quasiblack hole configuration where $R / M \simeq 1.0$.

\subsection{Charge of a relativistic incompressible star as a function of the energy density}

In Fig. 5, the charge to mass relation $Q / M$ is plotted against the energy density $\rho_{0}$, for some values of the central pressure, and for the charge fractions $\alpha=0.5$ (top panel) and $\alpha=0.99$ (bottom panel). The red point in the upper left corner of the bottom panel indicates the quasiblack hole configuration $(Q / M=1.0)$. It is seen that in all cases the ratio $Q / M$ decreases as $\rho_{0}$ is increased. For sufficiently small central pressures, each curve presents a plateau in the low density region. The width of the plateau is larger (and higher) for high charge fractions, and, as the energy density grows, the ratio $Q / M$ decays very rapidly. The minimum value of $Q / M$ is around the corresponding value of $\alpha$. For instance, in the $\alpha=0.5$ case (top panel) the charge to mass ratio decreases from approximately $Q / M \simeq 0.7$ at low energy densities to approximately $Q / M=0.5$ at $\rho_{0}=1.0 \times 10^{20}\left[\mathrm{~kg} / \mathrm{m}^{3}\right]$. This value is found for $\rho_{0} \gtrsim 10^{3} p_{c}$. Another important feature is that the fraction $Q / M$ increases with the central pressure. From this we see that the larger the central pressure, the larger the amount of charge the star admits. In fact, it is seen from the $\alpha=0.99$ case (bottom panel) that there are incompressible charged stars very close to the quasiblack hole configuration, i.e., with $Q / M \simeq 1.0$.

The amount of charge supported by the incompressible spheres can also be seen in Fig. 6, which shows the ratio $Q / M$ as a function of $\alpha$ for two values of the energy density $\rho_{0}$. In this figure, the central pressure is $1.0 \times 10^{18}\left[\mathrm{~kg} / \mathrm{m}^{3}\right]$ and the energy densities are $1.0 \times 10^{13}\left[\mathrm{~kg} / \mathrm{m}^{3}\right]$ and $1.0 \times 10^{20}\left[\mathrm{~kg} / \mathrm{m}^{3}\right]$. The point in red in this figure, as in the bottom panel of Fig. 5. indicates that for $\alpha=1.0$ it is found $Q / M=1.0$.

\subsection{The interior pressure of a charged incompressible star}

We now study the behavior of the pressure inside these charged incompressible spheres, i.e., we study $p(r)$. Figure 7 shows a few particular cases of $p(r)$. The panel at the top is for the energy density $\rho_{0}=10^{13}\left[\mathrm{~kg} / \mathrm{m}^{3}\right]$, the one in the middle is for $\rho_{0}=10^{18}\left[\mathrm{~kg} / \mathrm{m}^{3}\right]$, and the panel at the bottom is for $\rho_{0}=10^{20}\left[\mathrm{~kg} / \mathrm{m}^{3}\right]$. Each panel shows the results for two values of the charge fraction, $\alpha=0.0$ and $\alpha=0.99$, and two central pressures $p_{c}=$ $10^{13}\left[\mathrm{~kg} / \mathrm{m}^{3}\right]$ and $p_{c}=10^{18}\left[\mathrm{~kg} / \mathrm{m}^{3}\right]$. As expected, the pressure within the star decreases monotonically from the center toward the surface of the object in all the analyzed cases. For stars with central pressure smaller than the energy density the pressure varies very slowly with the radial coordinate, going to zero with a fast rate just close to the surface of the star. The general behavior is that of a star with constant pressure from the center up to very close to the surface. On the other hand, stars
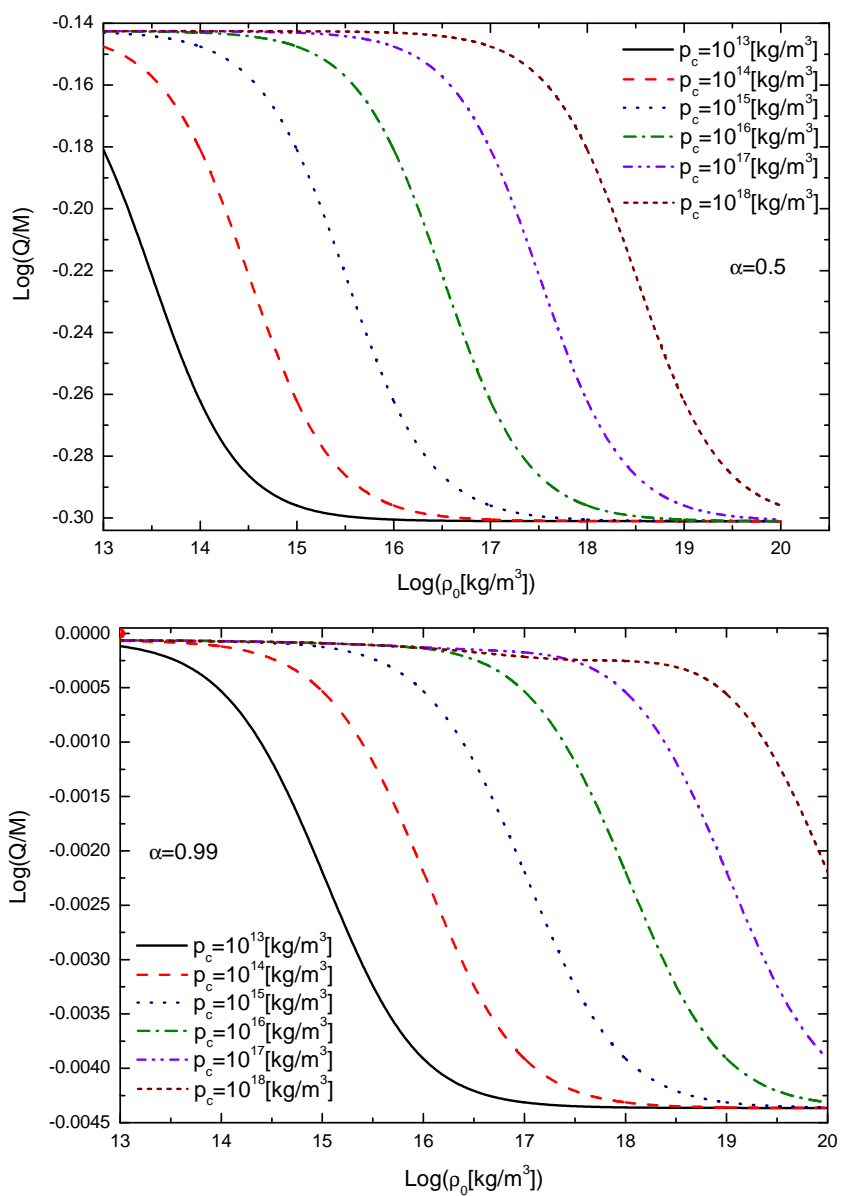

FIG. 5: The ratio $Q / M$ of the incompressible charged spheres as a function of the energy density $\rho_{0}$ for six values of the central pressure $p_{c}$ as indicated. The top panel is for $\alpha=0.5$ and the bottom panel for $\alpha=0.99$. The red point in the figure at the bottom indicates the quasiblack hole configuration where $Q / M \simeq 1.0$. 


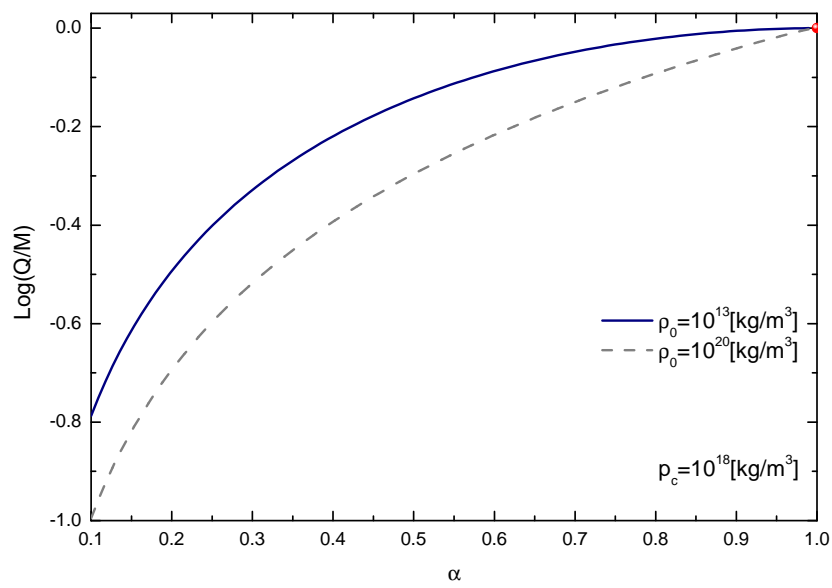

FIG. 6: The ratio $Q / M$ of the incompressible charged spheres as a function of the charge fraction $\alpha$ for the central pressure $1.0 \times 10^{18}\left[\mathrm{~kg} / \mathrm{m}^{3}\right]$ and two values of the energy density as indicated. The red point indicates the quasiblack hole limit where $Q / M \simeq 1.0$.

with central pressure larger than the energy density have a larger pressure gradient. In particular, in the limit of a very large central pressure, the pressure has to decrease abruptly with the radial coordinate to reach vanishing values at the surface. It is clearly observed that for fixed energy density and charge fraction, the radius of a given star increases with the central pressure. In general, the increase of the central pressure implies an increase of the size $R$ of the equilibrium solutions and an increase in the mass $M$, while the resulting star becomes more compact, in the sense that $R / M$ approaches unity. From Figs. 3 and 7 it is seen that more compact stars have larger radii and larger mass and charge.

\section{THE ELECTRIC INTERIOR SCHWARZSCHILD LIMIT OF A CHARGED RELATIVISTIC INCOMPRESSIBLE STAR AND THE BUCHDAHL-ANDRÉASSON LIMIT}

The solid black line in Fig. 8 gives the ratio $R / M$ as a function of $Q / R$ found by means of the TOV equation, for the lowest energy density and the largest central pressure used in the present analysis, i.e., for $\rho_{0}=$ $1.0 \times 10^{13}\left[\mathrm{~kg} / \mathrm{m}^{3}\right]$ and $p_{c}=1.0 \times 10^{18}\left[\mathrm{~kg} / \mathrm{m}^{3}\right]$, respectively. Numerically, this discrepancy of $10^{5}$ between $p_{c}$ and $\rho_{0}$ simulates the electric interior Schwarzschild limit, as this happens for $p_{c} / \rho_{0} \rightarrow \infty$. Thus, the solid black line gives the electric interior Schwarzschild limit $p_{c} / \rho_{0} \rightarrow \infty$. For $Q / R=0$, i.e., for the interior Schwarzschild solution, one gets the original interior Schwarzschild limit of $R / M=9 / 4=2.25$ [1]. In the other extreme, for $Q / R=1$, one gets the quasiblack hole $R / M=1$. In between these charges the values of $R / M$ for this limit are displayed in the curve. As noted before, numerically we work with $\alpha=0.99$ rather than $\alpha=1$, and so our results give rather $Q / M \simeq 1.0, Q / R \simeq 1.0$, and $R / M \simeq 1.0$.
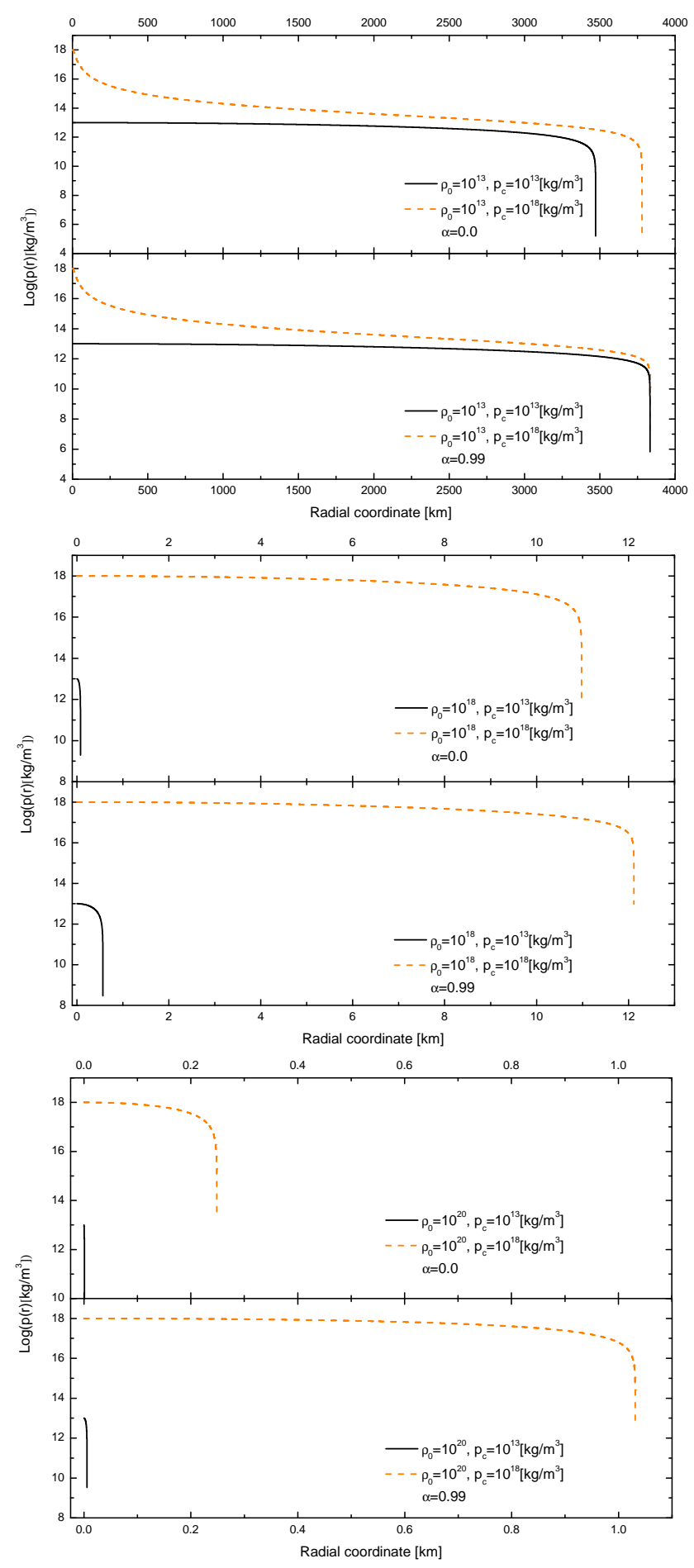

FIG. 7: The figure shows the pressure $p$ of the fluid as a function of the radial coordinate $r$. The panel at the top is for the energy density $\rho_{0}=10^{13}\left[\mathrm{~kg} / \mathrm{m}^{3}\right]$, the one in the middle is for $\rho_{0}=10^{18}\left[\mathrm{~kg} / \mathrm{m}^{3}\right]$, and the panel at the bottom is for $\rho_{0}=10^{20}\left[\mathrm{~kg} / \mathrm{m}^{3}\right]$. Each panel displays $p(r)$ for two values of charge fraction, 0.5 and 0.99 and for two central pressures $p_{c}=10^{13}$ and $p_{c}=10^{18}\left[\mathrm{~kg} / \mathrm{m}^{3}\right]$.

One should also compare the electric interior Schwarzschild limit with the Buchdahl-Andréasson limit. The Buchdahl limit [6] a limit of limits, is obtained by 


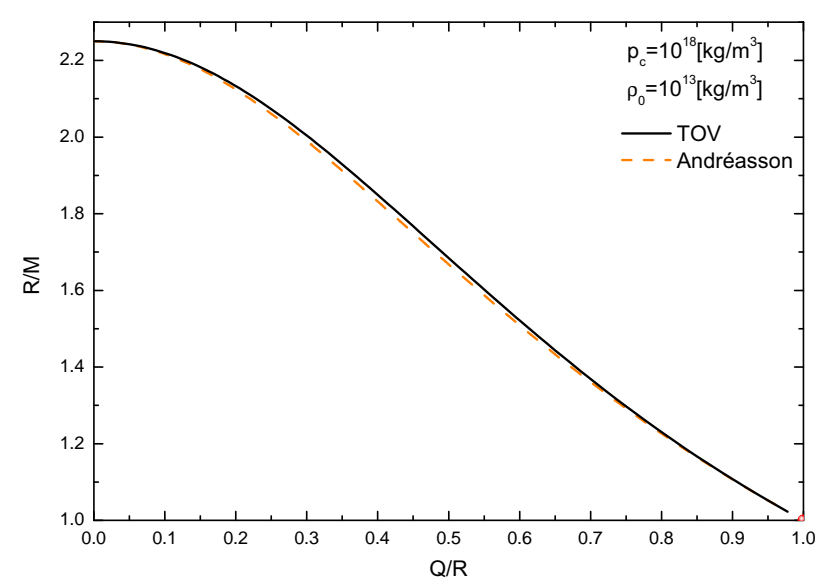

FIG. 8: The solid black line shows the electric interior Schwarzschild limit, i.e., the ratio $R / M$ versus the ratio $Q / R$ for very large central pressures, here for $\rho_{0}=1.0 \times 10^{13}\left[\mathrm{~kg} / \mathrm{m}^{3}\right]$ and $p_{c}=1.0 \times 10^{18}\left[\mathrm{~kg} / \mathrm{m}^{3}\right]$. The charge fraction varies from $\alpha=0.0$ to $\alpha=1$ (numerically, the maximum value of $\alpha$ we have managed to achieve was $\alpha=0.99$ ). The line is found using the TOV equation for these specific stars. The red dashed line gives the Buchdahl-Andréasson limit, i.e., the values found using the relation $R / M=9 /\left(1+\sqrt{1+3 Q^{2} / R^{2}}\right)^{2}$. The two curves coincide in the boundary points, namely, $Q / R=0$ and $Q / R=1$, but not in the intermediate points. The Buchdahl-Andréasson curve bounds the electric interior Schwarzschild limit curve and is indeed a limit of limits. The point in red, $Q / R=1$, represents the quasiblack hole limit.

imposing that the density of the star should be nonincreasing with radius $r$, the pressure is isotropic, and a few other reasonable assumptions. For stars with nonzero electrical charge there is the Buchdahl-Andréasson limit, a generalized Buchdahl limit found by Andréasson 14 by imposing other conditions, such as $p+2 p_{T} \leq \rho$, where $p$ and $p_{T}$ are the radial and tangential pressures, respectively, together with some other reasonable physical conditions. The Buchdahl-Andréasson bound is [14])

$$
\frac{R}{M} \geq \frac{9}{\left(1+\sqrt{1+3 Q^{2} / R^{2}}\right)^{2}}
$$

where the inequality gives the Buchdahl-Andréasson limit. For $Q=0$, Eq. 19 gives the Buchdahl bound $R / M \geq 9 / 4$, of course. In Fig. 8 the red dashed line is the Buchdahl-Andréasson limit. It is clearly seen in Fig. 8 , that there are two points where the two lines coincide, namely, $Q / R=0$ and $Q / R=1$. Indeed, for $\alpha=0$, i.e. $Q / R=0$, both the original interior Schwarzschild limit and the Buchdahl limit coincide, giving $R / M=9 / 4$. For $\alpha=1$, i.e., $Q / R=1$, both curves give $R / M=1$, i.e., both limits yield the quasiblack hole solution. On the other hand, it is also clearly seen in Fig. 8 that although the lines are near each other, they do not coincide, i.e., the electric interior Schwarzschild and the BuchdahlAndréasson limits are generically different. Undoubtedly, the values of $R / M$ shown by the solid line are very close but always larger than those shown by the dashed line.
This has been substantiated by analytical calculations valid up to order $Q^{2} / R^{2}$ on the interior Schwarzschild limit for these stars, and it points to the fact that the Buchdahl-Andréasson limit is indeed a limit of limits. As noted in [14], the $\rho=$ constant electric solutions discussed in [13] do not saturate the bound. In our work, we also find that our $\rho=$ constant electric solutions, although close, do not saturate the bound. Configurations that do saturate the Buchdahl-Andréasson bound are self-gravitating electrically charged shells 14. It will be interesting to investigate whether there are other configurations that saturate the bound.

\section{QUASIBLACK HOLE LIMIT OF A CHARGED RELATIVISTIC INCOMPRESSIBLE STAR}

An important point here is to investigate whether one finds configurations close to the quasiblack hole configuration or not. In the analysis we have found that the limit of the quasiblack hole configuration is reached for values of the central pressure much greater than the energy density and for large charge fractions.

It can be seen from Figs. 3, 4, 5, and 6 that for some values of the energy density, central pressure, and charge fraction, the relations $R / M$ and $Q / M$ reach unity. This limit appears when the central pressure is much larger than the energy density. In this instance, the parameters that best show these results are $\rho_{0}=1.0 \times 10^{13}\left[\mathrm{~kg} / \mathrm{m}^{3}\right]$, $p_{c}=1.0 \times 10^{18}\left[\mathrm{~kg} / \mathrm{m}^{3}\right]$, and $\alpha=0.99$. In this case, the quantities $M, R$, and $Q$ are such that $R / M=1.02268$ and $Q / M=0.999853$. These values can be interpreted as giving $R \simeq M \simeq Q$, indicating that this charged star is close to the quasiblack hole configuration.

In order to show that there is indeed a quasiblack hole in this limit, we analyze also the behavior of the metric functions, $A(r)$ and $B(r)$, as proposed in [28] (see also [29, 30]).

We plot $1 / A(r)$ versus the radial coordinate in Fig. 9 for the energy density $\rho_{0}=1.0 \times 10^{13}\left[\mathrm{~kg} / \mathrm{m}^{3}\right]$, the central pressure $p_{c}=1.0 \times 10^{18}\left[\mathrm{~kg} / \mathrm{m}^{3}\right]$, and the charge fraction $\alpha=0.99$. Observe that the function $1 / A(r)$ starts at unity at $r=0$ (no conical singularity) and decreases with the increasing of the radial coordinate, reaching its minimum value at the star's surface $r=R$. The value of $1 / A(r)$ at the surface of the star is approximately zero, actually it is $2.25942 \times 10^{-4}$. This vanishingly small value of $1 / A(r=R)$ indicates that the solution is close to a quasiblack hole configuration [28].

We plot the metric potential $B(r)$ as a function of the radial coordinate in Fig. 10 for the same values of energy density, pressure, and charge fraction as in the case of the function $1 / A(r)$. Once the values for the total mass $M$, radius $R$ of the star, and total charge $Q$ are already known, $B(r)$ is obtained by numerically integrating the differential equation (13) from the surface to the center of the star. The results show that the function $B(r)$ assumes values close to zero in the 


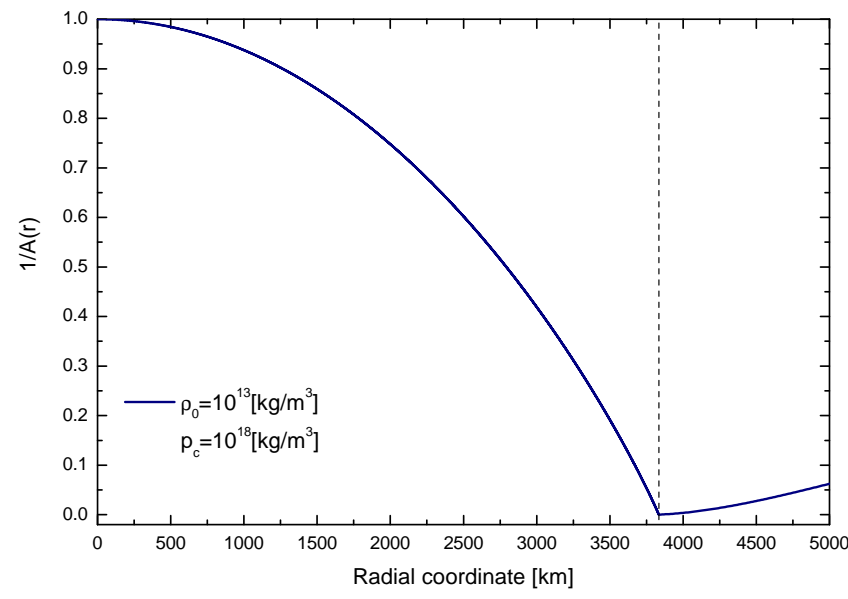

FIG. 9: The inverse of metric function $A(r)$ as a function of the radial coordinate for the quasiblack hole configuration. The parameters used are $\rho_{0}=1.0 \times 10^{13}\left[\mathrm{~kg} / \mathrm{m}^{3}\right]$, $p_{c}=1.0 \times 10^{18}\left[\mathrm{~kg} / \mathrm{m}^{3}\right]$, and charge fraction $\alpha=0.99$. The vertical dashed line indicates the surface of the star.

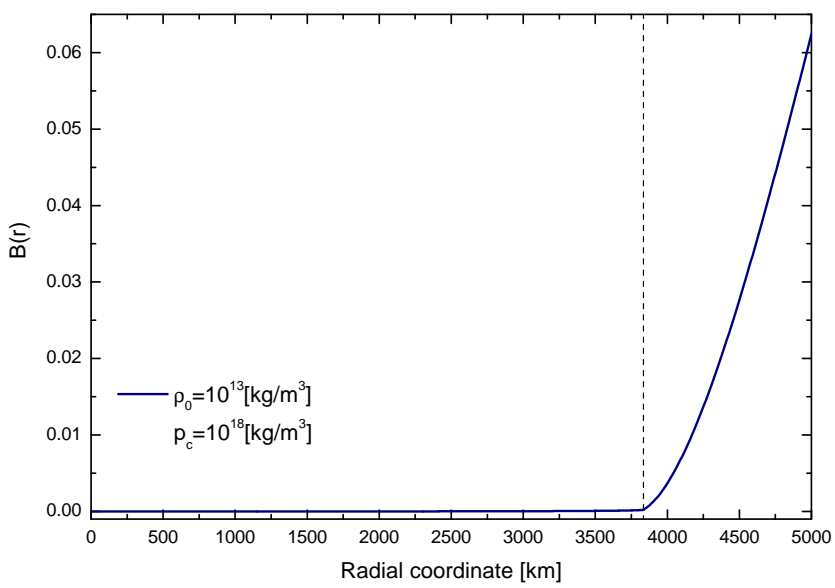

FIG. 10: The metric function $B(r)$ as a function of the radial coordinate for the quasiblack hole configuration. The parameters used are $\rho_{0}=1.0 \times 10^{13}\left[\mathrm{~kg} / \mathrm{m}^{3}\right], p_{c}=1.0 \times 10^{18}\left[\mathrm{~kg} / \mathrm{m}^{3}\right]$, and charge fraction $\alpha=0.99$. The vertical dashed line indicates the surface of the star.

whole interior region, confirming that this is on the verge of being a quasiblack hole solution 28. The value of $B(r)$ increases albeit slowly with the radial coordinate, reaching its maximum interior value at the surface of the star $(r=R)$. This value of $B(R)$ coincides with the minimum value of the function $A^{-1}(R)$, i.e., $B(R)=2.25942 \times 10^{-4}$. The equality between $B(r)$ and $A^{-1}(r)$ at $r=R$ satisfies the boundary conditions, namely, $B(R)=A^{-1}(R)=1-\frac{2 M}{R}+\frac{Q^{2}}{R^{2}}$. This comes from the junction conditions which impose continuity of the metric functions.

The event and the Cauchy horizons of a ReissnerNordström spacetime are given by the solutions of the equation $B(r)=0$, i.e., $r_{ \pm} \equiv M \pm \sqrt{M^{2}-Q^{2}}$, respectively. Then, since the mass, the radius, and the charge of the star are very close to each other, i.e., $R \simeq M \simeq Q$, the event and the Cauchy horizons obey $r_{ \pm} \simeq M$, with $R \gtrsim r_{+}$. This indicates that the solution is a regular static configuration. Since $R \simeq r_{+}$, we see that the boundary of the star approaches its own gravitational radius, i.e., a quasiblack hole with pressure is close to being reached.

An important quantity is the redshift at the surface of the system, i.e., how the light frequency of a wave emitted from the system's surface is redshifted away when it arrives at infinity. We have investigated numerically the behavior of the redshift for the charged incompressible stars presented, comparing the charged and uncharged cases for a few central pressures and central densities. The results are as expected from previous studies [28] (see also [25, 26]). In the quasiblack hole limit, the redshift at the surface of the stars is indefinitely large. However, numerically we were able to find values of the order of 100 , i.e., the larger ratio between the frequency of a flash of light emitted at the surface of the star compared to the observed frequency at spatial infinity we found numerically was of about 100 (the low ratio is due to numerical convergence problems in the limit of large charge fractions). In Fig. 11, the redshift function $B(R)^{1 / 2}-1$ at the quasiblack hole's surface is displayed for $0<\alpha<1$.

Quasiblack hole behavior has been also found in [8 10] for an incompressible fluid, where different equations for the charge density have been considered. Other works where quasiblack holes have been found are 23 27, to name a few.

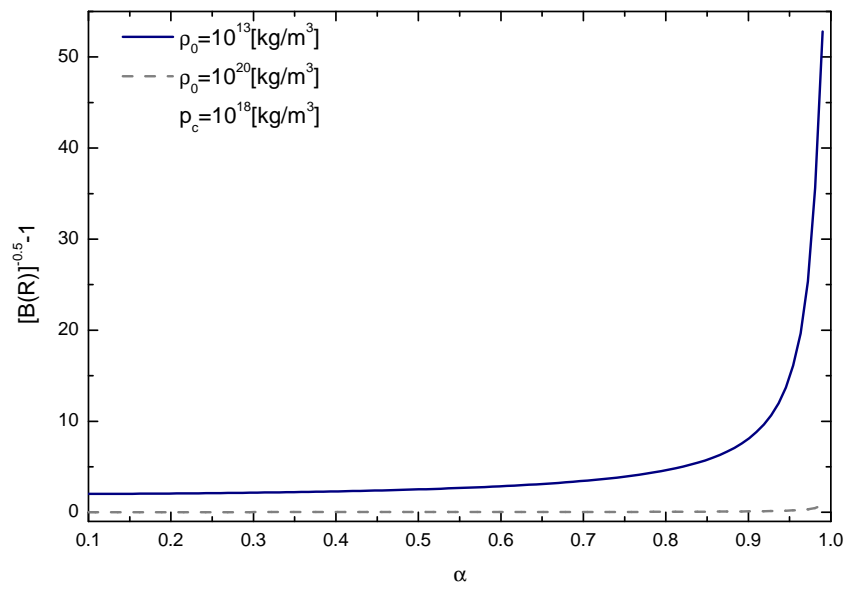

FIG. 11: The redshift function $B(R)^{1 / 2}-1$ at the surface of the quasiblack hole as a function of the charge fraction for the central pressure $p_{c}=1.0 \times 10^{18}\left[\mathrm{~kg} / \mathrm{m}^{3}\right]$ and two values of the energy density is displayed. The charged fraction $\alpha$ is varied from 0.1 to 0.99 .

\section{CONCLUSIONS}

In this work we have studied stars made of an incompressible fluid, i.e., a fluid with constant energy density $\rho_{0}$, and with an electrical charge distribution $\rho_{e}$ proportional to $\rho_{0}, \rho_{e}=\alpha \rho_{0}$, with $\alpha$ a number between 0 and 
1. The analyzed configurations have spherical symmetry whose exterior spacetime is described by the ReissnerNordström metric. The configurations were analyzed for different values of the energy density, central pressure and charge fraction. We have found that the electric interior Schwarzschild limit does not saturate the compactness Buchdahl-Andréasson bound, except in the border solutions $Q / M=0$ and $Q / M=1$. For the highest value of the charge fraction that still yields reliable numerical results, i.e., $\alpha=0.99$, and for a central pressure much larger than the energy density, we showed that there is a highly compact configuration with $R \simeq r_{+}$, i.e., a configuration on the verge of becoming a quasiblack hole.

Our results supplement the results of several previous works. In relation to the Buchdahl-Andréasson bound, as remarked in 14, the work 13 presents that electric stars with $\rho=\rho_{0}$ and $\rho_{0}$ a constant do not saturate the Buchdahl-Andréasson bound. Surely, the set of solutions found in [8 10, which include the solution discussed in [13, does not saturate the Buchdahl-Andréasson bound. In addition, analytical calculations, valid up to order $Q^{2} / R^{2}$, on the stars we have presented here also show that the Buchdahl-Andréasson bound is not saturated. In relation to quasiblack holes, on one hand, our work shows that the results found in [8 10] are robust. In [810] it was considered incompressible electrically charged fluids $\rho=\rho_{0}$ and $\rho_{0}$ a constant, but with a charge distribution that differs from ours, namely, $q(r)=Q(r / R)^{n}$ for some exponent $n \geq 3$ in [8, 9], or a more intricate distribution [10]. Numerical star solutions were found and it was shown that configurations with boundaries as close as one wants to their own gravitational radius are possible. I.e., quasiblack holes also appear in [8 [10]. On the other hand, our previous work [24] analyzes with care compressible configurations of stars with an equation of state $p=\omega \rho^{\gamma}$, for some constant $\omega$ and exponent $\gamma$, and also discusses the incompressible $\gamma \rightarrow \infty$ limit. In this work 24, the name Buchdahl limit was used for what we have called here more appropriately the interior Schwarzschild limit. Furthermore, the results presented here are also related to those displayed in [23] for the Guilfoyle exact configurations [17 which have a Cooperstock-de la Cruz-Florides equation of state [15, 16] and a different equation for the charge distribution. In brief, for a wide range of parameters, the structure of the charged stars change slightly when one changes the equations of state. However, not all equations of state have a quasiblack hole limit. An example of an equation of state that does not yield stars with a quasiblack hole limit is a polytropic equation of state, see e.g. [19] and [24].

\section{Acknowledgments}

JPSL and VTZ thank Francisco Lopes and Gonçalo Quinta for conversations and collaboration on the analytical calculations for the interior Schwarzschild limit. JDVA thanks Coordenação de Aperfeiçoamento de Pes- soal de Nível Superior - CAPES, Brazil, for a grant. VTZ would like to thank Conselho Nacional de Desenvolvimento Científico e Tecnológico - CNPq, Brazil, for grants, and Fundação de Amparo à Pesquisa do Estado de São Paulo for a grant (Processo 2012/08041-5). JPSL thanks the Fundação para a Ciência e a Tecnologia of Portugal FCT for support, Projects No. PTDC/FIS/098962/2008 and No. PEst-OE/FIS/UI0099/2011. JPSL and VTZ thank the Observatório Nacional do Rio de Janeiro - ON, Brazil, for hospitality.

\section{Appendix A: Equation of structure in dimensionless form}

For the numerical calculations it is convenient to write the equations of structure in a dimensionless form. We then introduce a rescaled radial coordinate $\varepsilon$ through the equation

$$
r=\frac{\varepsilon}{\sqrt{4 \pi \rho_{0}}},
$$

where we have made $G=1$ and $c=1$. In addition, new variables $\mu(\varepsilon), \theta(\varepsilon)$, and $\kappa(\varepsilon)$ are defined in terms of $m(r), p(r)$, and $q(r)$, respectively, by

$$
\begin{aligned}
m(r) & =\frac{\mu(\varepsilon)}{\sqrt{4 \pi \rho_{0}}}, \\
p(r) & =p_{c} \theta(\varepsilon), \\
q(r) & =\frac{\varepsilon^{2} \kappa(\varepsilon)}{\sqrt{4 \pi \rho_{0}}},
\end{aligned}
$$

where $\rho_{0}$ and $p_{c}$ represent the energy density and central pressure of the star, respectively. Now, in terms of the new variables $\mu, \theta$, and $\kappa$, Eqs. (8), 12, and (14) in dimensionless form become

$$
\begin{aligned}
& \frac{d \kappa}{d \varepsilon}=-\frac{2 \kappa}{\varepsilon}+\frac{\alpha}{\sqrt{1-\frac{2 \mu}{\varepsilon}+\varepsilon^{2} \kappa^{2}}} \\
& \frac{d \mu}{d \varepsilon}=\varepsilon^{2}+\frac{\alpha \varepsilon^{3} \kappa}{\sqrt{1-\frac{2 \mu}{\varepsilon}+\varepsilon^{2} \kappa^{2}}}, \\
& \frac{d \theta}{d \varepsilon}=-\varepsilon\left(\theta+\rho_{0} p_{c}^{-1}\right)\left(\frac{p_{c} \rho_{0}^{-1} \theta-\kappa^{2}+\frac{\mu}{\varepsilon^{3}}}{1-\frac{2 \mu}{\varepsilon}+\varepsilon^{2} \kappa^{2}}\right) \\
&+\frac{\alpha \rho_{0} p_{c}^{-1} \kappa}{\sqrt{1-\frac{2 \mu}{\varepsilon}+\varepsilon^{2} \kappa^{2}}},
\end{aligned}
$$

where we have also used relation 16 written in the new variables. This set of coupled differential equations, (A5)-A7), is solved to get the equilibrium solutions. The boundary conditions adopted in the center of the star, i.e., at $\varepsilon=0$, are $\kappa(0)=0, \mu(0)=0$, and $\theta(0)=1$.

Once the functions $\mu(\varepsilon)$ and $\kappa(\varepsilon)$ have been obtained by means of numerical integration, the metric potential 
$A(\varepsilon)$ is obtained from its definition, Eq. 111, which in the dimensionless variables reads

$$
A^{-1}=1-\frac{2 \mu}{\varepsilon}+\varepsilon^{2} \kappa^{2},
$$

while the metric potential $B(\varepsilon)$ is determined by integration of Eq. 13,

$$
\frac{d B}{d \varepsilon}=2 \varepsilon B\left(\frac{p_{c} \rho_{0}^{-1} \theta-\kappa^{2}+\frac{\mu}{\varepsilon^{3}}}{1-\frac{2 \mu}{\varepsilon}+\varepsilon^{2} \kappa^{2}}\right),
$$

where again we have replaced the original variables $r$, $q(r), m(r)$, and $p(r)$, by the respective dimensionless quantities $\varepsilon, \kappa(\varepsilon), \mu(\varepsilon)$, and $\theta(\varepsilon)$.

The integration of equations $\mathrm{A} 5 \mathrm{~A}$ - 7 comes to a halt at the point where the pressure $\theta$ reaches zero value, finding thus the value of $\varepsilon$ at the surface of the star, $\varepsilon=\varepsilon_{s}$. The corresponding value of the radial coordinate is extracted and the radius of the sphere is obtained from the relation $R=\frac{\varepsilon_{s}}{\sqrt{4 \pi \rho_{0}}}$. The other physical quantities, such as the mass $M$ and the charge $Q$, are calculated from the relations $M \equiv m(R)=\frac{\mu\left(\varepsilon_{s}\right)}{\sqrt{4 \pi \rho_{0}}}$ and $Q \equiv q(R)=\frac{\varepsilon_{s}^{2} \kappa\left(\varepsilon_{s}\right)}{\sqrt{4 \pi \rho_{0}}}$, respectively.
[1] K. Schwarzschild, "Über das Gravitationsfeld eines Kugel aus inkompressibler Flüssigkeit nach der Einsteinschen Theorie", Sitzungsberichte der Königlich Preussischen Akademie der Wissenschaften (Berlin) 1, 424, 1916; K. Schwarzschild, "On the gravitational field of a sphere of incompressible fluid according to Einstein's theory", arXiv:physics/9912033 (translation by S. Antoci).

[2] R. C. Tolman, "Static solution of Einstein's field equation for spheres of fluid", Phys. Rev. 55, 364 (1939).

[3] J. R. Oppenheimer and G. Volkoff, "On massive neutron cores", Phys. Rev. 55, 374 (1939).

[4] G. M. Volkoff, "On the Equilibrium of Massive Spheres", Phys. Rev. 55, 413 (1939).

[5] C. W. Misner, "Gravitational collapse", in Astrophysics and General Relativity, Brandeis University Summer School in Theoretical Physics, edited by M. Chrétien, S. Deser, and J. Goldstein (Gordon and Breach, New York 1968).

[6] H. A. Buchdahl, "General relativistic fluid spheres", Phys. Rev. 116, 1027 (1959).

[7] J. Bekenstein, "Hydrostatic equilibrium and gravitational collapse of relativistic charged fluid balls", Phys. Rev. D 4, 2185 (1971).

[8] F. de Felice, Y.-Q. Yu, and J. Fang, "Relativistic charged spheres", Mon. Not. R. Astron. Soc. 277, L17 (1995).

[9] F. de Felice, S.-M. Liu, and Y.-Q. Yu, "Relativistic charged spheres: II. Regularity and stability", Classical Quantum Gravity 16, 2669 (1999); arXiv:gr-qc/9905099.

[10] P. Anninos and T. Rothman, "Instability of extremal relativistic charged spheres", Phys. Rev. D 65, 024003 (2001); arXiv:gr-qc/0108082.

[11] Y.-Q. Yu and S.-M. Liu, Commun. Theor. Phys. 33, 571 (2000); arXiv:gr-qc/9904050

[12] C. G. Böhmer and T. Harko, "Minimum mass-radius ratio for charged gravitational objects", Gen. Relativ. Gravit. 39, 757 (2007); arXiv:gr-qc/0702078.

[13] A. Giuliani and T. Rothman, "Absolute stability limit for relativistic charged spheres", Gen. Relativ. Gravit. 40, 1427 (2008); arXiv:0705.4452 [gr-qc].

[14] H. Andréasson, "Sharp bounds on the critical stability radius for relativistic charged spheres", Commun. Math. Phys. 288, 715 (2009); arXiv:0804.1882 [gr-qc].

[15] F. I. Cooperstock and V. de la Cruz, "Sources for the Reissner-Nordström metric", Gen. Relativ. Gravit. 9, 835 (1978).

[16] P. S. Florides, "The complete field of charged perfect fluid spheres and of other static spherically symmetric charged distributions", J. Phys. A 16, 1419 (1983).

[17] B. S. Guilfoyle, "Interior Weyl-type solutions to the Einstein-Maxwell field equations", Gen. Relativ. Gravit. 31, 1645 (1999); arXiv:gr-qc/9906089

[18] J. L. Zhang, W. Y. Chau, and T. Y. Deng, "The influence of a net charge on the critical mass of a neutron star", Astrophys. Space. Sci. 88, 81 (1982).

[19] S. Ray, A. L. Espíndola, M. Malheiro, J. P. S. Lemos, and V. T. Zanchin, "Electrically charged compact stars and formation of charged black holes", Phys. Rev. D 68, 084004 (2003); arXiv:astro-ph/0307262

[20] C. Ghezzi, "Relativistic structure, stability, and gravitational collapse of charged neutron stars", Phys. Rev. D 72, 104017 (2005); arXiv:gr-qc/0510106.

[21] B. B. Siffert, J. R. de Mello, and M. O. Calvão, "Compact charged stars", Braz. J. Phys. 37, 609 (2007).

[22] D. Logoteta and I. Bombaci, "Quark deconfinement transition in neutron stars with the field correlator method", Phys. Rev. D 88, 063001 (2013); arXiv:1309.0096 [nuclth].

[23] J. P. S. Lemos and V. T. Zanchin, "Quasiblack holes with pressure: Relativistic charged spheres as the frozen stars", Phys. Rev. D 81, 124016 (2010).

[24] J. D. V. Arbañil, J. P. S. Lemos, and V. T. Zanchin, "Polytropic spheres with electric charge: compact stars, the Oppenheimer-Volkoff and Buchdahl limits, and quasiblack holes", Phys. Rev. D 88, 084023 (2013); arXiv:1309.4470 [gr-qc].

[25] W. B. Bonnor and S. B. P. Wickramasuriya, "Are very large gravitational redshifts possible?", Mon. Not. R. Astron. Soc. 170, 643 (1975).

[26] J. P. S. Lemos and E. Weinberg, "Quasiblack holes from extremal charged dust", Phys. Rev. D 69, 104004 (2004); arXiv:gr-qc/0311051

[27] K. A. Bronnikov, J. C. Fabris, R. Silveira, and O. B. Zaslavskii, "Dilaton gravity, (quasi)black holes, and scalar charge" (2014), arXiv:1312.4891 [gr-qc].

[28] J. P. S. Lemos and O. B. Zaslavskii, "Quasiblack holes: definition and general properties", Phys. Rev. D 76, 084030 (2007); arXiv:0707.1094 [gr-qc].

[29] J. P. S. Lemos and O. B. Zaslavskii, "Quasiblack holes with pressure: General exact results", Phys. Rev. D 82, 024029 (2010); arXiv:1004.4651 [gr-qc].

[30] J. P. S. Lemos, "Black holes and quasiblack holes: Some history and remarks", Uch. Zap. Kazan. Univers. 153, 
215 (2011); arXiv:1112.5763 [gr-qc]. 